\title{
DSM-5 ist da, wann kommt die ICD-11?
}

\author{
Mit der finalen Fassung der ICD-11 wird frühestens im \\ Jahr 2015 gerechnet. Bis dahin werden aber voraussicht- \\ lich noch mehrere Korrekturdurchläufe erforderlich sein.
}

Auch auf dem EPA-Kongress wurden die vorgesehenen Änderungen im DSM-5 - dessen finale Fassung zu der Zeit noch nicht veröffentlich war - kontrovers diskutiert. Das in Europa gängige internationale Klassifikationssystem der Weltgesundheitsorganisation, die ICD-10 (International Classification of Diseases) einschließlich seines umfangreichen psychiatrischen Teils, befindet sich immer noch im Revisionsprozess. Frühestens 2015 wird eine finale Fassung der ICD-11 vorliegen. Bis dahin muss voraussichtlich noch viel Arbeit in diverse Korrekturdurchläufe investiert werden. Im Rahmen des EPA-Kongresses gab Professor Wolfgang Gaebel, Düsseldorf, einen Überblick über den aktuellen Stand der Entwicklung des Revisionsprozess der ICD10. „Der klinische Nutzen ist das wichtigste organisatorische Prinzip“, so Gaebel. Es gehe nicht darum, neue Diagnosen zu schaffen, sondern um eine größere Exaktheit in der Diagnostik.
In Bezug auf das Kapitel der psychischen Störungen seien zwar in manchen Bereichen Angleichungen zwischen ICD-11 und DSM-5 zu erwarten. Auf der anderen Seite zeichne sich bereits jetzt ab, dass es bei einigen der aufzunehmenden psychischen Störungen und ihren Diagnosekriterien auch Unterschiede zwischen den beiden Klassifikationssystem geben könnte.

Bei der künftigen Klassifikation psychotischer Störungen in ICD-11 ist - wie auch in DSM-5 - die Einführung neuer Verlaufskriterien geplant. Die ersten Vorschläge zur Revision der Klassifikationskriterien für die die Schizophrenie in ICD-11 sehen vor, den Bereich „F2 Schizophrenie, schizotypische und wahnhafte Störungen " umzubenennen in Schizophrenie-Spektrum- und andere primär psychotischen Störungen. Auf die traditionellen Subtypen der Schizophrenie soll verzichtet werden. Außerdem wird empfohlen, den Einschluss kognitiver oder funktioneller Beeinträchtigungen als obligatorische Diagnosekriterien zu vermeiden.

Abdol A. Ameri, freier Medizinjournalist

Symposium "Schizophrenia Disorder and other primary psychosis according to ICD-11", 21. EPA, Nizza, 6. -9.4.2013

\section{Psychische Gesundheit - Strategien für Europa}

\author{
Das Europäische Parlament hat umfangreiche Projekte \\ und Strategien ins Leben gerufen, um die negativen Kon- \\ sequenzen psychischer Krankheiten einzudämmen und \\ die psychische Gesundheit der Bevölkerung zu fördern.
}

Psychische Störungen sind die führende Ursache für chronische Erkrankungen und Behinderung in der EU. Darüber hinaus sind sie mit einer rasch zunehmenden Prävalenz assoziiert. Schon heute erleidet jeder vierte EU-Bürger mindestens einmal im Leben eine ernsthafte psychische Erkrankung. Jedes Jahr verüben knapp 60.000 Menschen in der EU Suizid häufig infolge einer Depression. Menschen mit psychischen Störungen wiederum werden stigmatisiert, diskriminiert und sozial ausgeschlossen. Die gesundheitsökonomischen Belastungen durch psychische Krankheiten wie Schizophrenie, affektive Störungen, Angst, Panik, Depression, Alkohol- und Substanzmissbrauch sind für die Mitgliedsstaaten enorm. Erschwert werde die Situation zusätzlich durch die ökonomische Krise, berichtete Michael Hübel, Generaldirektion Gesundheit und Konsumentenschutz, Europäische Kommission. Denn einerseits gefährden die finanziellen Sorgen der Menschen in den besonders schwer betroffenen Mitgliedsstaaten die psychische Gesundheit; zugleich geraten auch die Gesundheitsbudgets unter zunehmenden Druck. Das Europäische Parlament hat daher im Rahmen des "Joint Action on Mental Health and WellBeing" umfangreiche Projekte und Strategien ins Leben gerufen, um die negativen Konsequenzen psychischer Krankheiten einzudämmen und die psychische Gesundheit der Bevölkerung zu fördern, so Hübel. Die neue von der EU unterstützte und von der Universität Lissabon koordinierte Aktion startete im Februar 2013 und läuft bis Januar 2016. Im Zentrum dieser Strategien stehen unter anderem präventive Maßnahmen und die Förderung der psychischen Gesundheit am Arbeitsplatz und in der Schule. Als besonders wichtig werden die Vermeidung von Suiziden, die Entwicklung und der frühzeitige Einsatz diagnostischer Testverfahren sowie eine gezielte integrierte Behandlung erachtet. Außerdem werde der Entstigmatisierung psychischer Krankheiten ein hoher Stellenwert zugeschrieben.

\section{EU-Experten sehen Schlüsselrolle der Psychiatrie}

In Bezug auf die Versorgung psychisch Kranker habe die Psychiatrie eine Schlüsselrolle, konstatierte der EU-Gesundheitsexperte. Dabei gehe es aber auch um die Umsetzung von Patienten-orientierter Therapiemodellen und um sektorenübergreifende Maßnahmen zur Frühintervention. Außerdem müsse der enge Zusammenhang zwischen psychischer und physischer Gesundheit mehr berücksichtigt werden. Im Hinblick auf die häufigen körperlichen Komorbiditäten von Patienten mit psychischen Erkrankungen sei es aber auch eine Aufgabe des Psychiaters, die Verbindung zu anderen medizinischen Fachgebieten herzustellen, um die diagnostische Abklärung und adäquate Behandlung dieser Begleiterkrankungen zu gewährleisten.

Abdol A. Ameri, freier Medizinjournalist

Symposium "Strategies to diminish the burden of mental health disorders", 21. EPA, Nizza, 6.-9.4.2013 